\section{Commentary: Coronary artery bypass grafting after acute myocardial infarction: Is timing of surgery a relevant factor?}

\author{
Vito Domenico Bruno, MD, PhD
}

How important is the coronary artery bypass grafting (CABG) timing after an acute myocardial infarction (MI)? For many years we have been discussing this topic, ${ }^{1}$ but we still do not have a predominant recommendation on when to operate in a patient after an MI, and in most of the cases we make ad hoc decisions. Moreover, confusing messages are coming from the scientific literature. Although some studies would support a delayed surgery, ${ }^{1,2}$ other authors would not agree with this. This is the case of the study by Bianco and colleagues ${ }^{3}$ in this issue of the Journal. In a retrospective single-center analysis, the authors have reviewed 8 years of experience in this type of clinical scenario, concluding that there are no significant differences between fast ( $<24$ hours after MI) and delayed surgery ( $\geq 24$ hours after MI) in terms of major adverse cardiovascular events (MACCE) and mortality. Bianco and colleagues ${ }^{3}$ reached their conclusions using a stabilized inverse probability treatment weighting (IPTW), which is one of the propensity score methods used to diminish the impact of preoperative biases or differences that are often present in retrospective analysis. At first sight, it seems clear that after IPTW, the 2 groups have similar results; therefore, the conclusions are supported by this type of comparative analysis. But I believe a word of caution should be given. As shown in Table E1, ${ }^{3}$ the preoperative characteristics of the patients before IPTW were significantly different in

\footnotetext{
From the Bristol Medical School, Translational Health Science, University of Bristol, Bristol, United Kingdom.

Disclosures: Author has nothing to disclose with regard to commercial support.

Received for publication Dec 21, 2019; accepted for publication Dec 23, 2019; available ahead of print Jan 23, 2020.

Address for reprints: Vito Domenico Bruno, MD, PhD, Bristol Medical School, Translational Health Sciences, University of Bristol, Research Floor Level 7, Bristol Royal Infirmary, Upper Maudlin St, BS2 8HW Bristol, UK (E-mail: Vito. D.Bruno@bristol.ac.uk).

J Thorac Cardiovasc Surg 2021;161:2065-6 0022-5223/ $\$ 36.00$

Crown Copyright $(\subset) 2020$ Published by Elsevier Inc. on behalf of The American Association for Thoracic Surgery

https://doi.org/10.1016/j.jtcvs.2019.12.083
}

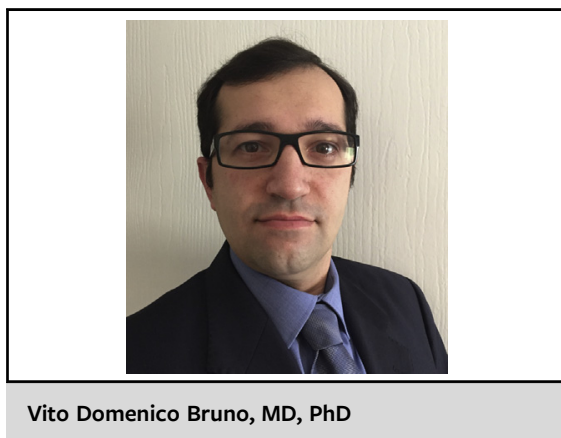

CENTRAL MESSAGE

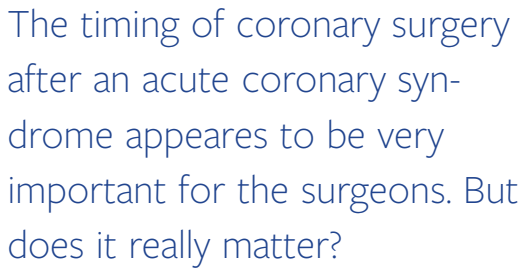

many aspects. As you would expect, before IPTW, in the less than 24 hours group there was a higher number of patients undergoing operation in an emergency setting or with a salvage operation, but this difference disappeared after IPTW; a similar observation applies to the Society of Thoracic Surgeons score that is higher in the less than 24 hours group before IPTW. Given the nature of the MI and the several different aspects that characterize its clinical behavior, an oversimplification of these patients might represent a mistake when reporting clinical outcomes. Surely the propensity score methods are a sensible way to minimize preoperative differences that could have an impact on the outcome, but certain characteristics are intrinsic to the nature of MI and should not be ignored. In this case, the event of interest is not the surgery itself, but rather the MI and perhaps the IPTW could have been based on pre-MI variables only. We should not forget that some of these patients, and especially those undergoing operation less than 24 hours after the MI, undergo an operation as a last option, and this is reflected in Table E1 in Bianco and colleagues' article. ${ }^{3}$ Not surprisingly, in the overall population, the operative mortality was almost double in the fast surgery group, but this difference was mitigated after risk adjustments with IPTW: The danger here is that the IPTW might have actually reduced the negative role of those patients who were at higher risk for surgery (ie, unstable patients) and therefore improving the outcome of the less than 24 hours group. A review published in $2013^{4}$ found that only $39 \%$ of the previously published articles demonstrated an association between timing of surgery and clinical outcomes, whereas preoperative characteristics such 
as the urgency of the operation or the presence of unstable angina were consistently associated with the results: The authors of this review wrote that "time by itself is an unreliable criterion to decide on surgical revascularization, but the acuity of illness is a major determinant of outcomes." 4 Despite this, Bianco and colleagues ${ }^{3}$ report on a large population and provide many helpful insights. An important feature of this article is represented by the reported longterm MACCE that has been approached with a competing-risk analysis and shows that the timing is not associated with the incidence of MACCE, whereas other factors such as diabetes, peripheral vascular disease, chronic obstructive pulmonary disease, prior heart failure, and age were significant predictors of MACCE readmissions. Again, it seems that the most significant predictor of this event is represented by the overall clinical status of the patients rather than the timing of the surgery. At the same time, the authors show that the long-term survival is not affected by the timing of surgery. With a remarkable 5 -year survival of $78.75 \%$ in the 24 hours or more group and an $83.47 \%$ in the less than 24 hours group, we can certainly support the role of surgery in these patients, but it has to be noted that the Cox proportional hazard model demonstrates that the 24 hours or more group was associated with a significant reduction in mortality. Another important part of this study is related to the stratification of the patients based on the type of MI (ST-segment elevation myocardial infarction vs non-ST segment elevation myocardial infarction), and it is interesting to note in
Supplemental Table 3 that the short-term mortality rate was higher in the less than 24 hours group, but this difference was more prominent in the presence of a non-ST segment elevation myocardial infarction. Overall, the authors have certainly provided an important retrospective analysis of the role of CABG after MI, but are we really convinced that these patients should receive an operation at less than 24 hours from their acute event? The answer to this question would have a significant impact on our practice, and I believe further studies would be needed before confirming this thesis. As Bianco and colleagues ${ }^{3}$ advise at the end of their article, "the presence of comorbidities plays an important role in determining CABG outcomes in the setting of MI." I am in agreement with this statement. The indication for surgery should still be related to the specific clinical condition of each specific patient, and every case should be carefully evaluated before proceeding to surgery, irrespective of the timing of the MI.

\section{References}

1. Lee DC, Oz MC, Weinberg AD, Windsor T. Appropriate timing of surgical intervention after transmural acute myocardial infarction. J Thorac Cardiovasc Surg. 2003;125:115-20.

2. Weiss ES, Chang DD, Joyce DL, Nwakanma LU, Yuh DD. Optimal timing of coronary artery bypass after acute myocardial infarction: a review of California discharge data. J Thorac Cardiovasc Surg. 2008;135:503-11.

3. Bianco V, Kilic A, Gleason TG, Aranda-Michel E, Wang Y, Navid F, et al. Timing of coronary artery bypass grafting after acute myocardial infarction may not influence mortality and readmissions. J Thorac Cardiovasc Surg. 2021;161:2056-64.e4.

4. Caceres M, Weiman DS. Optimal timing of coronary artery bypass grafting in acute myocardial infarction. Ann Thorac Surg. 2013;95:365-72. 